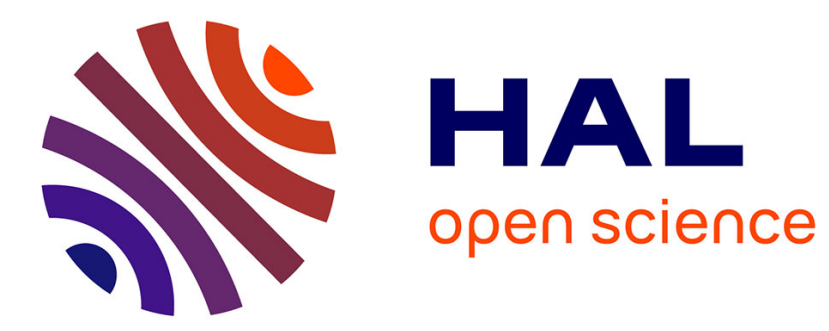

\title{
Apis mellifera hemocytes generate increased amounts of nitric oxide in response to wounding/encapsulation
}

Pedro Negri, Silvina Quintana, Matias Maggi, Nicolas Szawarski, Lorenzo Lamattina, Martin Eguaras

\section{- To cite this version:}

Pedro Negri, Silvina Quintana, Matias Maggi, Nicolas Szawarski, Lorenzo Lamattina, et al.. Apis mellifera hemocytes generate increased amounts of nitric oxide in response to wounding/encapsulation. Apidologie, 2014, 45 (5), pp.610-617. 10.1007/s13592-014-0279-0 . hal-01234761

\section{HAL Id: hal-01234761 \\ https://hal.science/hal-01234761}

Submitted on 27 Nov 2015

HAL is a multi-disciplinary open access archive for the deposit and dissemination of scientific research documents, whether they are published or not. The documents may come from teaching and research institutions in France or abroad, or from public or private research centers.
L'archive ouverte pluridisciplinaire HAL, est destinée au dépôt et à la diffusion de documents scientifiques de niveau recherche, publiés ou non, émanant des établissements d'enseignement et de recherche français ou étrangers, des laboratoires publics ou privés. 


\title{
Apis mellifera hemocytes generate increased amounts of nitric oxide in response to wounding/encapsulation
}

\author{
Pedro Negri ${ }^{1,3,5}$, Silvina Quintana ${ }^{4}$, Matias MagGi ${ }^{1,3}$, Nicolas Szawarski ${ }^{1}$, \\ Lorenzo Lamattina ${ }^{2,3}$, Martin Eguaras ${ }^{1,3}$ \\ ${ }^{1}$ Laboratorio de Artrópodos, Facultad de Cs Exactas y Naturales, Universidad Nacional de Mar del Plata (UNMdP), \\ Mar del Plata, Argentina \\ ${ }^{2}$ Instituto de Investigaciones Biológicas-CONICET, Universidad Nacional de Mar del Plata, Mar del Plata, \\ Argentina \\ ${ }^{3}$ Consejo Nacional de Investigaciones Científicas y Técnicas (CONICET), Sarmiento, Argentina \\ ${ }^{4}$ Laboratorio de Biología Molecular, Fares Taie Instituto de Análisis, Mar del Plata, Argentina \\ ${ }^{5}$ Funes 3350, Facultad de Cs Exactas y Naturales, nivel 0, Mar del Plata PC 7600, Argentina
}

Received 7 November 2013 - Revised 3 February 2014 - Accepted 14 February 2014

\begin{abstract}
Apis mellifera populations are being threatened by several pathogens and parasitosis. Several authors have proposed that honey bee colonies may suffer from a compromised immune system leading to colony loss. This is why the study of $A$. mellifera immune system has become a topic of pressing concern. Nitric oxide (NO) is a signaling and immune effector molecule that has been proposed as a key molecule in invertebrate immunity, and that plays a part in A. mellifera cellular defenses. This paper deals with NO participation in the response to wounding/encapsulation challenge in A. mellifera fifth instar (L5) larvae. Challenging $A$. mellifera L5 larvae with nylon implants enhanced NO production and spreading in granulocytelike hemocytes and increased the number of this NO-producing hemocyte type. However, AmNOS expression levels were not influenced by the insult. These results reveal that NO participates in the wound healing/ encapsulation response as a signal molecule, possibly by the activation of a constitutively expressed AmNOS in honey bees.
\end{abstract}

Apis mellifera / nitric oxide / wounding / encapsulation

\section{INTRODUCTION}

Apis mellifera populations have been declining over the last years, and a current notion suggests that honey bee colonies may be suffering from a compromised immune system which could be associated to colony losses (Alaux et al. 2010).

Corresponding author: P. Negri, pedronegri1@yahoo.com.ar Manuscript editor: Klaus Hartfelder M. Eguaras and L. Lamattina contributed equally as senior authors.
Thus, the study of $A$. mellifera immune system has become a topic of great relevance in the last years. Nonetheless, most of the studies undertaken so far have centered on humoral and molecular approaches rather than on cellular response (Gätschenberger et al. 2013). The cellular immune response comprises wound healing, phagocytosis, nodulation, and encapsulation of the intruder (Gätschenberger et al. 2013). Among these, wound healing and encapsulation responses have been the less explored ones.

One of the main threats affecting A. mellifera colonies worldwide is the ectoparasitic mite Varroa destructor. This parasite feeds from A. mellifera 
larvae hemolymph diminishing its fitness due to blood extraction and virus vectorization, such as the Deformed Wing Virus (DWV) (Martin et al. 2012; Gätschenberger et al. 2013). To achieve long periods of hemolymph sucking, $V$. destructor secretes anticoagulant factors in its saliva that adversely affect the wound healing response of $A$. mellifera larvae (Richards et al. 2011). This anticoagulant effect is essential to the mite reproductive phase, since $V$. destructor broods are incapable of puncturing the larvae cuticle and need their mother to pierce it (Rosenkranz et al. 2010). All these facts support the notion that the wounds caused by $V$. destructor in A. mellifera larvae represent a crucial threat to be faced by the bee's immune response. Unfortunately, there is scant literature on the wound healing response of honey bees.

Nitric oxide (NO) is a highly reactive and unstable free radical gas produced by the oxidation of L-arginine to citrulline mediated by the enzyme NO synthase (NOS). In vertebrates, three main isoforms of this enzyme have been reported as follows: endothelial (eNOS), neuronal (nNOS), and cytokine inducible (iNOS). To date, just one NOS isoform has been found in each invertebrate species, with either a constitutive (signaling) or an inducible (toxic) role. In some cases, the same isoform has been found to play both roles (Rivero 2006). The easiness with which NO crosses the cell membranes allows for a very efficient response that is ideal for the transmission of cellular signals (Rivero 2006; Moreno-García et al. 2013). When produced in large quantities, NO becomes toxic because of its strong reactivity with oxygen and oxygen-related reactive intermediates, which release several toxic species with enzymatic and DNA-damaging properties. In this way, NO becomes toxic to many kinds of pathogens, including viruses, fungi, bacteria, and parasites, among them intracellular and extracellular protozoa and some metazoan parasites (Rivero 2006). But NO also plays a key part in the immune response, participating as a signaling molecule like, for instance, in the wound healing process (Moreno-García et al. 2013). Due to NO ubiquity and generality, this molecule has been proposed as a key molecule for future ecological immunologic studies (Rivero 2006; Moreno-García et al. 2013). Recently, we have reported that NO is produced after nonself recognition in A. mellifera hemocytes, and that it plays a part in hemocyte spreading (Negri et al. 2013).

Since NO participates in A. mellifera immune response (Negri et al. 2013), the wound healing response represents a definite challenge when it comes to confronting $V$. destructor. Given its contribution in the animal wound healing process, the aim of this work was to determine if NO was produced within hemocytes after an injury in A. mellifera. We could show that NO is produced in hemocytes of wounded larvae, and that the number of hemocyte types which produce NO increases after the wound challenge. This suggests that NO may play a role in the wound healing response in A. mellifera.

\section{MATERIALS AND METHODS}

\subsection{Insects}

Honey bees (A. mellifera) were reared in Langstroth hives located in Santa Paula experimental apiary in Ruta $226 \mathrm{~km} \mathrm{10,} \mathrm{Mar} \mathrm{del} \mathrm{Plata} \mathrm{city,} \mathrm{Buenos} \mathrm{Aires} \mathrm{province,}$ Argentina. Brood combs containing A. mellifera larvae of the first day of the fifth instar (L5) were taken to the laboratory where the experiments were conducted. The beehives belonged to a regional hybrid of $A$. mellifera (A. mellifera ligustica, A. mellifera mellifera). The colonies used were previously standardized for bee population, brood area, and honey and pollen storage. Each colony consist of 10 combs covered with adult bees, 6.5 combs with bee brood, and 3.5 combs with storage (honey and pollen).

\subsection{Nylon implant challenge assays}

To trigger a wound healing/encapsulation response (Wilson-Rich et al. 2008), nylon segments were implanted in L5 larvae. The implants consisted in a nylon thread ( $0.155 \mathrm{~mm}$ diameter) knotted on one end so that approximately $1 \mathrm{~mm}$ was introduced in the larva hemocele. Each larva was pinpointed using one pair of tweezers to immobilize the larva and another one to 
manipulate the implant and introduce it into the larva. Larvae were maintained in an incubator at $32-34{ }^{\circ} \mathrm{C}$ and $70 \%$ relative humidity. At $24 \mathrm{~h}$, the implants were removed and the larvae were used to evaluate gene expression or measure hemocyte behavior parameters (nitric oxide production, spreading, and types of adherent hemocytes attached to the glass bottom of the wells). Comparisons were conducted between the control groups (not implanted) and the implanted group (implanted with the nylon thread). The time chosen for the analysis $(24 \mathrm{~h})$ was based on previous observations were the total wound closure in a L5 larva was accomplished at about $30 \mathrm{~h}$ (data not shown).

\subsection{Hemolymph collection}

Hemolymph collection was performed as previously described by Negri et al. (2013). Briefly, insect blood was collected from fifth instar larvae by puncturing the soft cuticle with a sterile $30 \mathrm{G}$ needle. Before puncturing, the cuticle was surface sterilized with $70 \%$ ethanol. The hemolymph was collected with a micropipette and transferred to a microcentrifuge tube containing Excell405 insect cell culture media (Sigma) (1:10 ratio of hemolymph/media). Hemolymph solution was homogenized by soft pipetting into the sampling tube and then transferred to sterile 96-well glass bottom plates (NUNC, 96-well optical bottom plates, sterile) where all the hemocyte behavior parameters (NO production, spreading, and types of adherent hemocytes) were analyzed. Equal volumes of hemolymph were extracted from the control and the implanted larvae $(16 \mu l)$.

\subsection{Nitric oxide detection and quantification}

The endogenous synthesis of NO in living hemocytes was evidenced as in Negri et al. (2013) by means of the fluorescent probe DAF-FM DA (Molecular probes, Invitrogen) at a final concentration of $5 \mu \mathrm{M}$. This dye emits green fluorescence within cells after reacting with NO. To measure the nitric oxide production in the hemocytes, pictures were taken and the green fluorescence emitted by the cells was quantified using ImageJ (National Institute of Health, USA, public domain software). This has been proven to be a trustful proceeding which allows obtaining quality measure- ments for NO production within A. mellifera hemocytes cultured in vitro (Negri et al. 2013).

\subsection{Hemocyte spreading evaluation}

Cells that attached and spread upon stimulation with the glass surface were considered as immunerelated hemocytes. Hemocyte count was avoided by using a hemocytometer. This reduced the manipulation of the hemocytes analyzed in the assays. Cellular spreading of granulocyte-like and plasmatocyte-like hemocyte types was measured using ImageJ.

\subsection{Hemocyte types quantification}

To determine the effects of the nylon implants on hemocyte differentiation and/or discharge from the hematopoietic organs, the proportion of the different types of adherent hemocytes (which are immunerelated cells) was measured. The importance of counting the hemocytes attached to the glass bottom of the wells lies in that it allows selecting immunerelated live hemocytes (Negri et al. 2013). At the same time, given the fact that these cells were attached and spread in a linear relationship with the thin bottom of the wells resulting in cells dispersed over one plane of focus, comparisons could be made between several wells in regard to hemocyte types and quantities. The hemocyte type observed and counted was also in agreement with NO production.

\subsection{Gene expression analysis}

\subsubsection{RNA extraction, DNAse treatment, and reverse transcription}

Total RNA from A. mellifera L5 larvae was isolated using TRI-reagent (BioBasic Inc., USA), according to the manufacturer's protocol, and digested with DNAse I amplification grade (Invitrogen, Carlsbad, CA, USA) for $30 \mathrm{~min}$ at $37{ }^{\circ} \mathrm{C}$ to remove any contaminating genomic DNA (gDNA). The quantity of the resulting RNA was determined in a Rotor Gene 6,000 cycler using a Quant-i ${ }^{\text {TM }}$ RiboGreen ${ }^{\circledR}$ RNA assay kit (Invitrogen). Complementary DNA (cDNA) was synthesized using a reaction mixture containing $1 \mu \mathrm{g}$ of total RNA, random hexamers $(12 \mathrm{ng} / \mu \mathrm{L})$, and Moloney murine 
leukemia virus reverse transcriptase (Invitrogen), following the procedures suggested by the manufacturer. Negative controls, omitting the RNA or the reverse transcriptase, were included.

\subsection{Primers}

Primers were synthesized by Eurofins MWG Operon (Huntsville, AL, USA). Details of the primers are as follows: AmNOS: forward, 5'TCCACTCGCAGGTACTTTCC-3'; AmNOS: reverse, 5'-TCTGGAGGATCACCATTTCC-3' (Gregorc et al. 2012); $\beta$-actin: forward, 5'-ATGCCA ACACTGTCCTTTCTGG-3'; $\beta$-actin: reverse, 5'GACCCACCAATCCATACGGA-3' (Yang and CoxFoster 2005).

\subsection{RT-qPCR}

RT-qPCR reactions for AmNOS were run using EvaGreen as intercalating dye (KAPA FAST, Biosystems, Woburn, USA) and $2 \mu \mathrm{L}$ of cDNA sample in a final volume of $20 \mu \mathrm{L}$. The amplification and detection of the specific products were carried out using a Rotor Gene 6,000 cycler, with the following amplification conditions: $2 \mathrm{~min}$ at $95^{\circ} \mathrm{C}, 40$ cycles of $10 \mathrm{~s}$ at $95{ }^{\circ} \mathrm{C}, 15 \mathrm{~s}$ at $60{ }^{\circ} \mathrm{C}$, and $15 \mathrm{~s}$ at $72{ }^{\circ} \mathrm{C}$. After amplification, a melting curve analysis was performed, which resulted in a single product-specific melting curve. In all cases, the experiments were performed in triplicate. Negative controls for cDNA synthesis and qPCR procedures were included in all cases. The amplification efficiency was determined for each gene using tenfold dilutions of cDNA. Results are reported as the mean fold change of AmNOS transcription levels in implanted larvae over levels detected in nonimplanted larvae, which served as the control group during $24 \mathrm{~h}$ of wounding/encapsulation challenge.

The levels of AmNOS were studied, and $\beta$-actin was selected as a reference gene. $\beta$-actin cycle threshold $(\mathrm{Ct})$ values differed in less than $1 \mathrm{Ct}$ between controls and treated samples.

\subsection{Microscopy}

After being transferred to the 96-well plates, the mixtures containing A. mellifera hemolymph were observed using inverted microscopy. Hemocytes were examined by means of differential interference contrast (DIC) or epifluorescence (excitation at $480 \mathrm{~nm}$-emission at $520 \mathrm{~nm}$ ) microscopy. The microscope used was a Nikon Eclipse $T i$ inverted microscope using a $\times 60$ objective.

\subsection{Statistical analysis}

The relative expression analysis of the target gene AmNOS was performed using the Relative Expression Software Tool (REST, QIAGEN Inc., Valencia, CA, USA), which compares the expression of the target gene in a sample relative to the control, and tests the group differences for significance with a pairwise fixed reallocation randomization test. RTqPCR efficiency for each gene was determined by a linear regression model, according to the equation $E=$ 10 [-1/slope $]$.

To evaluate NO production and spreading in the NO-producing hemocytes, Mann-Whitney tests were performed (Figure 1), while a $t$ test was applied (Figure 1) to compare the number of NO-producing hemocytes between treatments. To analyze plasmatocyte-like hemocytes spreading, a MannWhitney test was conducted (Figure 3).

\section{RESULTS}

3.1. Nitric oxide is produced in $A$. mellifera L5 larvae hemocytes after nylon implant challenge

Nylon implants are considered as an immune challenge to trigger nonself recognition followed by encapsulation and wound healing in insect hemocytes (Wilson-Rich et al. 2008). Therefore, to assess the endogenous NO production in response to wounding and encapsulation, $A$. mellifera worker L5 larvae were challenged with nylon thread implants. The endogenous NO production of $A$. mellifera L5 larvae hemocytes was evaluated $24 \mathrm{~h}$ after the implants challenge using the fluorescent dye specific for NO detection DAF-FM DA. Nitric oxide production was analyzed immediately after hemolymph extraction. The green fluorescence within the cells was indicative of endogenous NO production. Nitric oxide production was detected in response to 

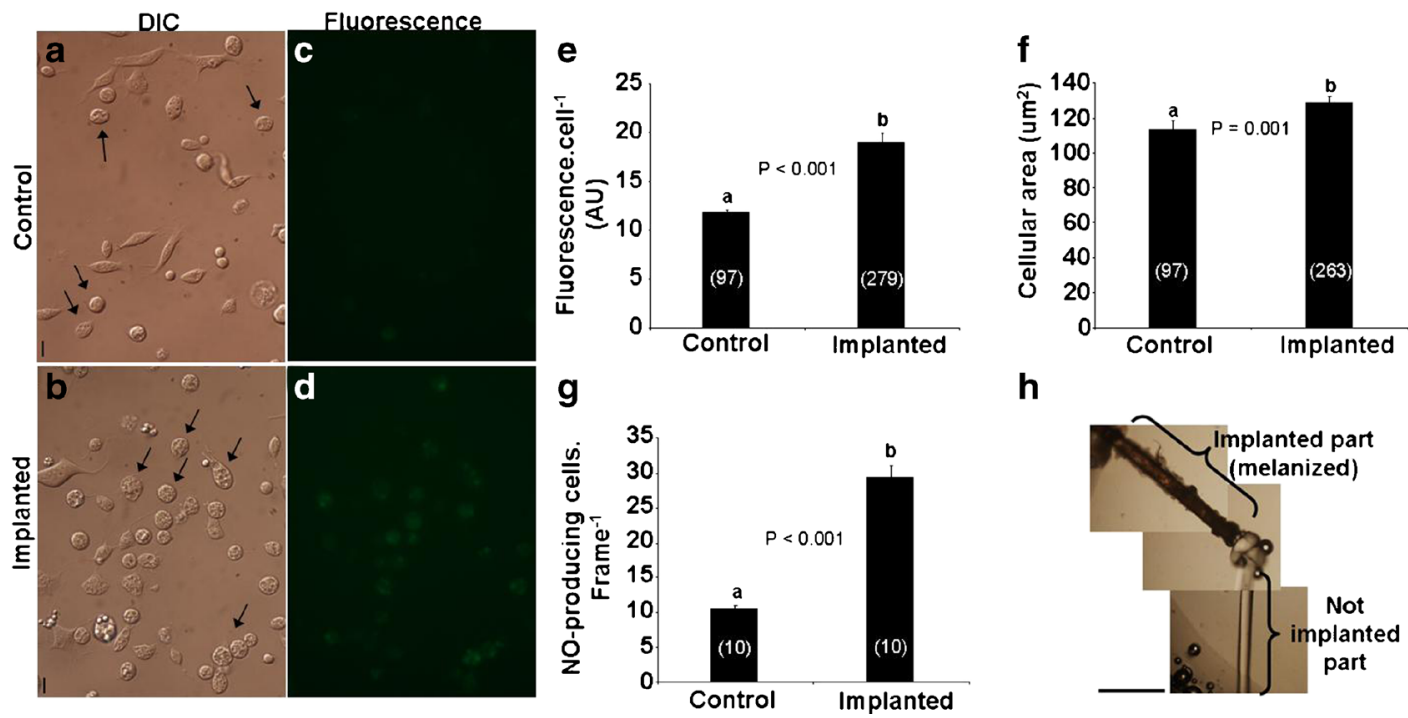

h

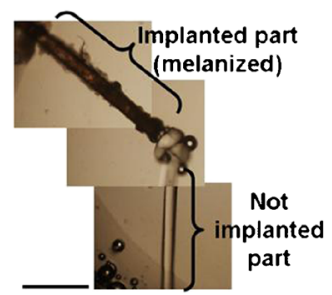

Figure 1. Apis mellifera hemocytes produce nitric oxide $(N O)$ in response to wound challenge. Honey bee fifth instar larvae (L5) were implanted (Implanted) or not implanted (Control) with a nylon thread. Twenty-four hours later, hemolymph was extracted from each larva, hemocytes were photographed under light $(\mathbf{a}, \mathbf{b})$ and epifluorescence $(\mathbf{c}$, d) microscopy; and NO production (e Mann-Whitney, $P<0.001$ ) and spreading (f Mann-Whitney, $P=0.001$ ) of the hemocytes were evaluated using ImageJ. Black arrows indicate the NO-producing hemocyte types. The number of NO-producing hemocytes per frame (frame $=3.10^{5} \mu \mathrm{m}^{2}$ ) was also compared between treatments $(\mathrm{g} t$ test, $P<0.001)$. Different letters mean significant differences between treatments. " $N$ " values are between parenthesis. Scale bar= $10 \mu \mathrm{m}$. A representative image of an encapsulated nylon implant is shown in $\mathbf{h}$.

wound challenge particularly within the same adherent-to-glass granulocyte-like hemocyte type as it was described by Negri et al. (2013) (Figure 1a-d). The hemocytes extracted from the larvae challenged with nylon implants (implanted) showed significantly more nitric oxide production than nonimplanted larvae (control) did (Figure 1e). In addition, granulocyte-like hemocytes from the implanted larvae appeared to be more spread as compared to the control cells (Figure 1f). Interestingly, hemolymph extracted from implanted larvae yielded significantly greater amounts of granulocyte-like hemocytes than hemolymph extracted from control larvae did (Figure 1g).

\subsection{Gene expression studies}

To determine whether wounding/encapsulation challenge had an effect on A. mellifera nitric oxide synthase (AmNOS) expression, the levels of AmNOS mRNA were quantified $24 \mathrm{~h}$ after the implantation of nylon threads in $A$. mellifera L5 larvae. AmNOS mRNA expression levels from implanted larvae were compared to the expression of AmNOS in control larvae (nonimplanted). No statistically significant differences $(P>0.05)$ were found for the expression levels of AmNOS mRNA in implanted when compared to control larvae (Figure 2).

\subsection{Plasmatocyte-like hemocytes from $A$.} mellifera $\mathbf{L} 5$ larvae also responded to nylon implant challenge but produced no NO at the time analyzed

Nitric oxide production was also evaluated in plasmatocyte-like hemocytes $24 \mathrm{~h}$ after the implant challenge. At this time, no differences were observed with respect to $\mathrm{NO}$ production comparing 


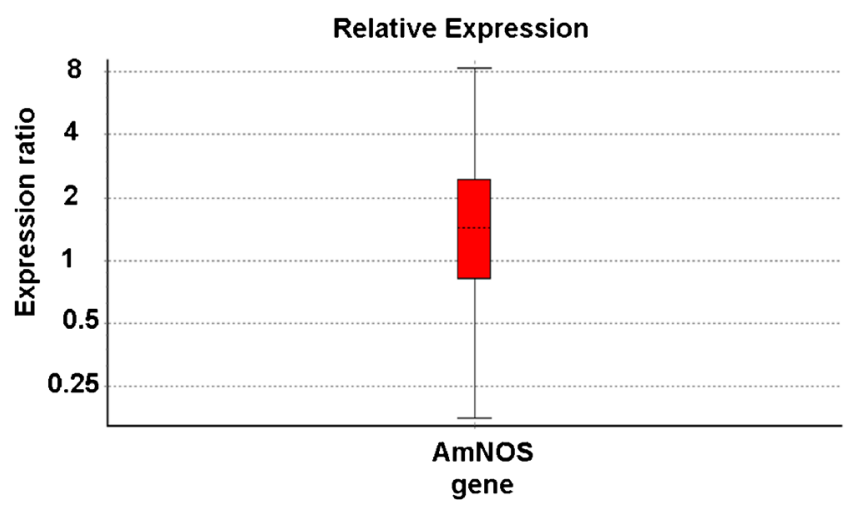

Figure 2. AmNOS relative expression levels of Apis mellifera nitric oxide synthase (AmNOS) in response to wounding/encapsulation challenge. These results are presented as a Whisker box plot. The graph illustrates the average of the expression ratios calculated for AmNOS gene in implanted larvae relative to the expression ratios for the same gene in the control larvae (considered as "1"). Three independent experiments were performed and a total of 18 larvae per treatment were analyzed.

hemocytes extracted from the implanted larvae to hemocytes from the control ones. Nevertheless, the plasmatocyte-like hemocytes from the implanted larvae were significantly more spread than the control ones were (Figure 3).

\section{DISCUSSION}

The recent decline in managed honey bee populations is of scientific, ecological, and economic concern, being partially attributed to honey bee parasites and related diseases
(Wagoner et al. 2013). It has been proposed that the diseases caused by infectious agents are a major selective pressure on honey bees. Finally, chronic increases in individual mortality and morbidity may lead directly to hive collapse or cause long-term reductions in hive-level fitness due to loss of productivity (Jefferson et al. 2013). This explains why the study of $A$. mellifera immune system has awoken a great interest in the last years. Even though several studies have addressed humoral and molecular approaches, more research regarding the cellular
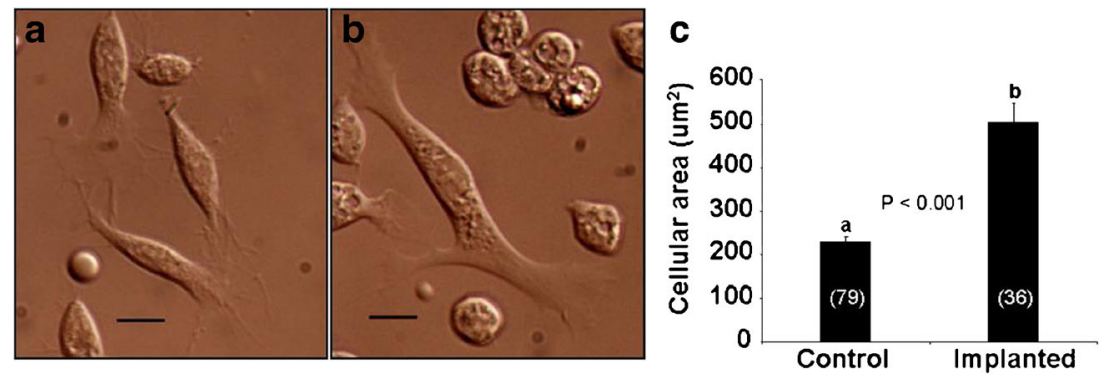

Figure 3. Enhanced spreading behavior of A. mellifera plasmatocytes after nylon implant insult. Honey bee fifth instar larvae (L5) were implanted (Implanted, b) or not implanted (Control, a) with a nylon thread. Twenty-four hours later, hemolymph was extracted from each larva, hemocytes were photographed under light microscopy (a, b), and spreading of the plasmatocytes was evaluated using ImageJ (c). Different letters mean significant differences between treatments. " $N$ " values are between parenthesis. Mann-Whitney, $P<0.001$ scale bar $=10 \mu \mathrm{m}$. 
responses of honey bees should be conducted (Gätschenberger et al. 2013). Our research effort is intended as a small contribution to improving the understanding of the wound healing/encapsulation response in $A$. mellifera.

In a previous report, we demonstrated that $\mathrm{NO}$ is produced by $A$. mellifera hemocytes in response to nonself recognition and that it plays a role in hemocyte spreading (Negri et al. 2013). Here, it is shown that a wounding/ encapsulation challenge, like the nylon implant, triggers NO production in the same $A$. mellifera hemocyte type described in Negri et al. (2013). Moreover, it is demonstrated that this enhancement is not observed in NO production, at least during the study period in the plasmatocyte-like hemocytes of $A$. mellifera L5 larvae. Nevertheless, plasmatocyte-like hemocytes were immune-activated by the implant challenge as evidenced by the enhancement of their spreading behavior. When challenging an insect with encapsulation, it is very important to observe the immune activation of plasmatocyte-like hemocytes (Strand 2008). These results suggest that NO signaling is hemocyte-specific; NO being produced and signaled through the granulocyte-like hemocytes described herein and previously by Negri et al. (2013). The signaling role exerted by NO in these different hemocyte type interactions merits further analysis.

The increase in NO production was not accompanied by an upregulation of AmNOS expression in the implanted larvae with respect to the control ones. Thus, the enhanced NO production noticed in the hemocytes extracted from the implanted larvae could result from NOS enzyme activation or derive from a nonenzymatic way of NO production (Park et al. 2013). Nevertheless, additional experiments should be carried out using extracted hemolymph rather than the entire larvae to analyze AmNOS gene expression in hemocytes. More experiments dealing with AmNOS expression in response to the wounding/encapsulation insult at times other than the time point analyzed here $(24 \mathrm{~h})$ should also be performed.
It is established that NOS enzymes found in invertebrates function either as an inducible or as a constitutive form depending on the insult (Rivero 2006). The upregulation of a NOS gene expression in insects has been associated to bacterial challenges (Hillyer and Estévez-Lao 2010; Davies and Dow 2009). In general, the presence of iNOS is linked to pathological conditions (Frank et al. 2002). On the contrary, the immune challenge performed here is aseptic. The implantation of sterilized nylon threads was used to trigger a wounding/encapsulation immune response in L5 A. mellifera larvae. These are multicellular immune responses characterized by the cooperation of many hemocytes where cellular signaling is imperative to succeed. The results provided herein suggest that NO plays a signaling role in the wounding/ encapsulation response in A. mellifera.

Interestingly, the nylon implants also induce greater amounts of the NO-producing granulocytes, suggesting that this type of hemocyte is crucial for the wound healing/encapsulation response. The mechanism that generates the large number of NO-producing hemocyte type opens new horizons for research. It could be ascribed to the release of this type of hemocytes from the hematopoietic organs or be the consequence of hemocyte differentiation in this hemocyte class.

Apart from cellular and humoral reactions, the activation of prophenoloxidase (proPO) by a serine protease cascade resulting in the transient synthesis of quinones and melanin is a prerequisite for the immune process of wound healing (Gätschenberger et al. 2013). Melanin pigmentation appears on the surface of the cuticle where the pathogen breaches the integument, as well as very near the surface of organisms that have invaded the host hemocoel (Nappi and Christensen 2005). Certain melanin intermediates interact with reactive intermediates of oxygen (ROI) and nitrogen (RNI) and enhance cytotoxic activity (Nappi and Christensen 2005). ROI and RNI generated during melanogenesis, along with pigment precursors, have been implicated in the killing of parasites by insects (Nappi and Christensen 2005). Thus, 
research advancing our understanding on the role of NO in the melanization response should be conducted to better describe the participation of $\mathrm{NO}$ as a signal molecule in A. mellifera immune response.

\section{ACKNOWLEDGMENTS}

This research was supported by PICT-2012-0594 granted by the "Agencia Nacional de Promoción Científica y Tecnológica" to M.E. The authors would like to thank CONICET and the UNMdP. Pedro Negri is a doctoral fellow from CONICET, Argentina.

Les hémocytes d'Apis mellifera produisent des quantités accrues d'oxyde nitrique en réponse à une blessure/encapsulation

Abeille domestique/larve/défense immunitaire/réaction de défense

Als Antwort auf eine Verwundungs/ Einschlussreaktion produzieren die Hämozyten von Apis mellifera erhöhte Mengen an Stickoxid

Apis mellifera/Stickoxid/Verwundung/ Einschlussreaktion

\section{REFERENCES}

Alaux, C., Ducloz, F., Crauser, D., Le Conte, Y. (2010) Diet effects on honey bee immunocompetence. Biol. Lett. 6, 562-565. doi:10.1098/rsbl.2009.0986

Davies, S., Dow, J. (2009) Modulation of epithelial innate immunity by autocrine production of nitric oxide. Gen. Comp. Endocrinol. 162, 113-121

Frank, S., Kämpfer, H., Wetzler, C., Pfeilschifter, J. (2002) Nitric oxide drives skin repair: novel functions of an established mediator. Kidney Int. 61, 882-888

Gätschenberger, H., Azzami, K., Tautz, J., Beier, H. (2013) Antibacterial immune competence of honey bees (Apis mellifera) is adapted to different life stages and environmental risks. PLoS ONE 8(6), e66415. doi:10.1371/journal.pone.0066415

Gregorc, A., Evans, J.D., Scharf, M., James, D., Ellis, J.D. (2012) Gene expression in honey bee (Apis mellifera) larvae exposed to pesticides and Varroa mites (Varroa destructor). J. Insect Physiol. 58, 1042-1049
Hillyer, J., Estévez-Lao, T. (2010) Nitric oxide is an essential component of the hemocyte-mediated mosquito immune response against bacteria. Dev. Comp. Immunol. 34, 141-149

Jefferson, J., Dolstad, H., Sivalingam, M., Snow, J. (2013) Barrier immune effectors are maintained during transition from nurse to forager in the honey bee. PLoS ONE 8(1), e54097. doi:10.1371/ journal.pone.0054097

Martin, S.J., Highfield, A.C., Brettell, L., Villalobos, E.M., Budge, G.E., Powell, M., Nikaido, S., Schroeder, D.C. (2012) Global honey bee viral landscape altered by a parasitic mite. Science 336, 1304

Moreno-García, M., Córdoba-Aguilar, A., Condé, R., Lanz-Mendoza, H. (2013) Current immunity markers in insect ecological immunology: assumed trade-offs and methodological issues. Bull. Entomol. Res. 103, 127-139

Nappi, A., Christensen, B. (2005) Melanogenesis and associated cytotoxic reactions: applications to insect innate immunity. Insect Biochem. Mol. Biol. 35, 443-459

Negri, P., Maggi, M., Correa-Aragunde, N., Brasesco, C., Eguaras, M., Lamattina, L. (2013) Nitric oxide participates at the first steps of Apis mellifera cellular immune activation in response to non-self recognition. Apidologie . doi:10.1007/s13592-0130207-8

Park, J.W., Piknova, B., Huang, P.L., Noguchi, C.T., Schechter, A.N. (2013) Effect of blood nitrite and nitrate levels on murine platelet function. PLoS ONE 8(2), e55699. doi:10.1371/journal.pone. 0055699

Richards, E., Jones, B., Bowman, A. (2011) Salivary secretions from the honey bee mite, Varroa destructor: effects on insect hemocytes and preliminary biochemical characterization. Parasitology 138, 602-608. doi: $10.1017 /$ S0031182011000072

Rivero, A. (2006) Nitric oxide: an antiparasitic molecule of invertebrates. Trends Parasitol. 22, 219-225

Rosenkranz, P., Aumeier, P., Ziegelmann, B. (2010) Biology and control of Varroa destructor. J. Invertebr. Pathol. 103, 96-119

Strand, M.R. (2008) The insect cellular immune response. Insect science 15, 1-14

Wagoner, K., Boncristiani, H., Rueppell, O. (2013) Multifaceted responses to two major parasites in the honey bee (Apis mellifera). BMC Ecology 13, 26

Wilson-Rich, N., Dres, D., Starks, P. (2008) The ontogeny of immunity: development of innate immune strength in the honey bee (Apis mellifera). J. Insect Physiol. 54, 1392-1399

Yang, X., Cox-Foster, D.L. (2005) Impact of an ectoparasite on the immunity and pathology of an invertebrate: evidence for host immunosuppression and viral amplification. PNAS 21, 7470-7475 